\title{
DIRECTIONAL GEODESIC ACTIVE CONTOURS
}

G. Gallego, J.I. Ronda

\author{
Grupo de Tratamiento de Imágenes \\ Universidad Politécnica de Madrid \\ 28040-Madrid, Spain
}

\author{
A. Valdés
Departamento de Geometría y Topología
Universidad Complutense de Madrid \\ 28040-Madrid, Spain
}

\begin{abstract}
We present a non-conformal metric that generalizes the geodesic active contours approach for image segmentation. The new metric is obtained by adding to the Euclidean metric an additional term that penalizes the misalignment of the curve with the image gradient and multiplying the resulting metric by a conformal factor that depends on the edge intensity. In this way, a closer fitting to the edge direction results. The provided experimental results address the computation of the geodesics of the new metric by applying a gradient descent to externally provided curves. The good performance of the proposed techniques is demonstrated in comparison with other active contours methods.
\end{abstract}

Index Terms - Active contour, calculus of variations, Riemannian metric, edge detection, image segmentation.

\section{INTRODUCTION}

In the context of active contours $[1,2]$, an initial curve is driven toward the boundaries (i.e., edges) of desired objects in the image by means of a PDE derived from the optimality conditions defined by an energy functional. Without considering a-priori information of the shape of the objects to be detected, energies can be broadly classified as edge-based [2,3] or region-based [4], although, in some cases, the divergence theorem blurs the line between them [5]. In edge-based approaches, the evolution of the curve (i.e. contour) is driven by an edge detector of the image. In region-based approaches, the evolution of the curve is governed by a flow that attempts to partition the image into statistically distinct regions. Edgebased energies depend on local features of the image, whereas region-based energies respond better to global characteristics.

Although the topic of active contours is well known, there is still room for improvement. Here, we focus our attention in the design of an edge-based energy that is motivated by the geometry of local goodness of fit of a contour to the edges of the objects in the image.

This work has been partially supported by the Ministerio de Ciencia e Innovación of the Spanish Government under project TEC2010-20412 (Enhanced 3DTV). G. Gallego is supported by a Marie-Curie COFUND grant.
In this paper we propose a technique to augment the conformal geodesic active contour (GAC) framework [2] with directional information by taking into account not only the magnitude but also the direction of the gradient of the image. The key point is the replacement of the conformal Euclidean arc-length with a non-conformal metric dependent on image information. This defines a Riemannian metric in the image domain, where the boundaries of the target objects to be segmented appear as closed geodesic curves. Related previous works on adding directional information are $[6,5,3]$.

The paper is organized as follows: In section 2, we motivate the design of a target energy functional based on a nonconformal arc-length. In section 3, we derive the curve evolution gradient flow to optimize the energy. We show the performance of the segmentation technique in section 4. Finally, we discuss the results and point out future research directions in section 5 .

\section{PROPOSED ENERGY FUNCTIONAL}

Our goal is to design an energy functional for the segmentation problem based on a geometric interpretation in a Riemannian framework. The target boundary of the object to be segmented is the minimizer of some anisotropic length measure that combines image information and curve regularization.

Given an image $I: \Omega \rightarrow \mathbb{R}$, where $\Omega$ is an open subset of $\mathbb{R}^{2}$, suppose that, at a point $\mathbf{x}=(x, y)^{\top} \in \Omega$ there is an edge, signaled by a large value of the magnitude of the image gradient vector field $\nabla I$ pointing in the direction perpendicular to the edge. Also, suppose that a regular curve $\mathbf{C}(p)=$ $(x(p), y(p))^{\top}$ passes through $\mathbf{x}$ with tangent and inward normal directions given by $\mathbf{T}=\mathbf{C}_{p} /\left\|\mathbf{C}_{p}\right\|$ (with $\left\|\mathbf{C}_{p}\right\| \neq 0 \forall p$ ) and $\mathbf{N}$, respectively (see Fig. 1). A word about notation: we use bold-face symbols such as $\mathbf{T}$ to represent vectors, typewriter fonts such as $\mathrm{G}$ to denote matrices, and, in general, subscripts to denote derivatives (e.g., $\mathbf{C}_{p}:=\partial \mathbf{C} / \partial p$ ).

We propose to replace the conformal arc-length $d s_{\phi}:=$ $\phi\left\|\mathbf{C}_{p}\right\| d p, \phi(\mathbf{x})$ being a positive differentiable function known as edge indicator [2], with $d s_{M}:=g^{1 / 2}\left(\mathbf{C}_{p}, \mathbf{C}_{p}\right) d p$, where $g$ defines a Riemannian metric on $\Omega$, hence it is a bilinear form $g(\mathbf{u}, \mathbf{v})=\mathbf{u}^{\top} \mathbf{G} \mathbf{v}$ with $\mathbf{G}=\left(g_{i j}\right)$ being a sym- 


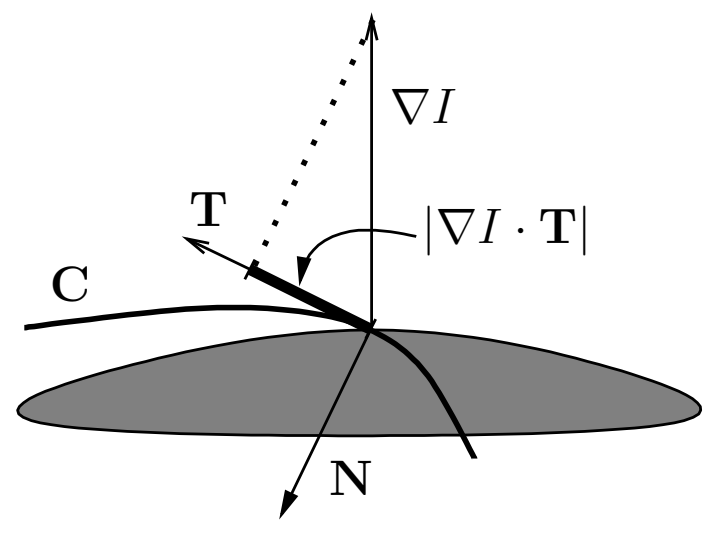

Fig. 1. Configuration of a curve passing near an edge in a grey-scale image $I$. The value $|\nabla I \cdot \mathbf{T}|$ is a possible measure of the misalignment between the contour and the edge. This quantity vanishes if the contour is perpendicular to the edge.

metric positive definite matrix. Furthermore, let $g^{1 / 2}=\phi f$, with $\phi(\mathbf{x})$ as before and $f=f\left(\nabla I, \mathbf{C}_{p}\right)$. The corresponding energy functional that measures the goodness of fit between the contour and the edge is

$$
\mathcal{L}_{M}=\int g\left(\mathbf{C}_{p}, \mathbf{C}_{p}\right)^{1 / 2} d p=\int \phi(\mathbf{C}(p)) f\left(\nabla I, \mathbf{C}_{p}\right) d p .
$$

The term $f$ penalizes the misalignment between the contour and the edge and it is clear that not any $f$ defines a Riemannian metric $g$. For adequate functions $f$, the minimizing curve can be interpreted as a geodesic for the corresponding metric. It is natural to use the inner product $\mathbf{C}_{p} \cdot \nabla I$ as a local measure of the misalignment. Therefore, we consider a weighted sum of such product and the usual Euclidean arclength:

$$
f^{2}=\mathbf{C}_{p} \cdot \mathbf{C}_{p}+\lambda q(\|\nabla I\|)\left(\mathbf{C}_{p} \cdot \frac{\nabla I}{\|\nabla I\|}\right)^{2},
$$

where $\lambda \geq 0$, and $q(r)$, with $r \geq 0$, is an increasing function such that $q(0)=0$. This yields a well-defined non-conformal Riemannian arc-length. Observe that $f$ favors the alignment of the curve perpendicular to the edge while $\phi$ enforces passing through zones with large gradient. The choice $q(r)=r^{2}$ leads to the simplified expression:

$$
f^{2}=\mathbf{C}_{p} \cdot \mathbf{C}_{p}+\lambda\left(\mathbf{C}_{p} \cdot \nabla I\right)^{2} .
$$

With this choice, the problem of finding the curve that minimizes the anisotropic length (1) becomes that of minimizing

$$
E(\mathbf{C})=\int_{C} \phi(\mathbf{C}(s)) \tilde{f}(\nabla I, \mathbf{T}) d s,
$$

where $s$ is the Euclidean arc-length parameter, $\left(d s=\left\|\mathbf{C}_{p}\right\| d p\right.$, hence $\mathbf{T}=\mathbf{C}_{s}$ ) and, according to (3), we define

$$
\tilde{f}(\nabla I, \mathbf{T}):=f\left(\nabla I, \mathbf{C}_{p}\right)\left\|\mathbf{C}_{p}\right\|^{-1}=\left(1+\lambda(\mathbf{T} \cdot \nabla I)^{2}\right)^{1 / 2} .
$$

Note that the energy (4) is "geometric" (or "intrinsic"), i.e., re-parameterization invariant, since it depends only on the curve $\mathbf{C}$ and not on the parameterization. This is a desired property to define a geometrically meaningful functional.

\subsection{Relations with other models}

From (3), it is straightforward to note that the Riemannian metric when $\phi=1$ is given by $\mathrm{G}=\mathrm{I}+\lambda \nabla I \nabla I^{\top}$ ( $\mathrm{I}$ being the identity matrix) since $f^{2}=\mathbf{C}_{p}^{\top}\left(\mathrm{I}+\lambda \nabla I \nabla I^{\top}\right) \mathbf{C}_{p}$. The term $\nabla I \nabla I^{\top}$ arises in the definition of the the structure tensor, or second-moment matrix, which describes the predominant directions of the gradient of the image $I$ in a neighborhood of a point. The structure tensor is commonplace in image processing and computer vision, for example, in the Harris and Stephens corner and edge detector [7], in image enhancement via anisotropic diffusion [8], etc.

The metric (3) can also be interpreted as the one given by the graph of $I$. The embedding of the scaled gray-level image $I$ as a surface $S(x, y)=(x, y, \sqrt{\lambda} I(x, y)) \subset \mathbb{R}^{3}$, yields the induced metric $\mathrm{G}$ (see $[9,10])$. Therefore, the goal of minimizing (4) with $\phi=1$ is that of finding geodesics in the manifold with the abovementioned metric determined by the image data $\sqrt{\lambda} I$.

The proposed model (1), (4) becomes the GAC model [2] in the particular case that $\lambda=0$ (i.e., $\tilde{f}=1$ ). Our model also differs from previous directional active contour approaches $[6,5,3]$. For example, the maximization of the energy in [5], $\int_{C}|\nabla I \cdot \mathbf{N}| d s$, is equivalent (by decomposition of $\nabla I$ in the Frenet frame $\{\mathbf{T}, \mathbf{N}\}$ ) to the minimization of $\int_{C}|\nabla I \cdot \mathbf{T}| d s=\int\left|\nabla I \cdot \mathbf{C}_{p}\right| d p$, which can be written as (4) with $g=\left|\nabla I \cdot \mathbf{C}_{p}\right|$, but this does not define a Riemannian metric since $\mathrm{G}=\nabla I \nabla I^{\top}$ is singular. Also, the anisotropic length in the Finsler active contour model [3] is less restrictive than the Riemannian model, hence it may not adopt the form (4). The choice between the adequacy of the Finsler or Riemannian framework depends on the application.

\section{ENERGY OPTIMIZATION}

To minimize the energy (4), we derive the steepest descent flow that will drive the evolution of the curve. For that, we compute the first variation of the energy.

Before we work out the general case let us discuss the simpler case where there is no conformal factor $(\phi=1)$. In this case, letting $E_{1}$ denote energy (4) with $\phi=1$, the functional gradient of $E_{1}$ in the usual $L^{2}$ inner product formulation [11] is given by the formula

$$
\nabla_{\mathbf{C}} E_{1}(s)=-\left(\tilde{f}^{-1} \mathbf{T}\right)_{s}-\lambda\left(\nabla I \cdot\left(\tilde{f}^{-1} \mathbf{T}\right)\right)_{s} \nabla I,
$$

which can be explicitly written in the normal direction:

$$
\nabla_{\mathbf{C}} E_{1}(s)=-\left(\kappa+\lambda(\nabla I \cdot \mathbf{N})(\nabla I \cdot \mathbf{T})_{s} \tilde{f}^{-2}\right) \tilde{f}^{-1} \mathbf{N}
$$


where $\kappa$ is the curvature and

$$
(\nabla I \cdot \mathbf{T})_{s}=\mathbf{T}^{\top} \nabla^{2} I \mathbf{T}+\kappa \nabla I \cdot \mathbf{N}
$$

with $\nabla^{2} I$ being the Hessian matrix of $I$. Also, an alternative formula in terms of the Laplacian of $I$ is $(\nabla I \cdot \mathbf{T})_{s}=(\Delta I-$ $\left.\mathbf{N}^{\top} \nabla^{2} I \mathbf{N}+\kappa \nabla I \cdot \mathbf{N}\right)$. Hence, the steepest descent flow in the iteration or "time" parameter $t$ is $\mathbf{C}_{t}=-\nabla_{\mathbf{C}} E_{1}(s)$, i.e.,

$$
\mathbf{C}_{t}=\left(\kappa+\lambda(\nabla I \cdot \mathbf{N})(\nabla I \cdot \mathbf{T})_{s} \tilde{f}^{-2}\right) \tilde{f}^{-1} \mathbf{N} .
$$

Observe that, if $\lambda=0$ (i.e., $\tilde{f}=1$ ) the energy $E_{1}$ becomes the Euclidean length of the curve and equation (8) coincides with the mean curvature flow $\mathbf{C}_{t}=\kappa \mathbf{N}$, which lacks directionality information of the edges in the image. Otherwise, the projections of the direction perpendicular to the edge $\nabla I$ onto the Frenet frame attached to the curve $\{\mathbf{T}, \mathbf{N}\}$ provide the building blocks of the curve flow with image-dependent directionality information.

Next, consider the case of a general $\phi$. In this case, the $L^{2}$ gradient of (4) is, in terms of the gradient (6),

$$
\begin{aligned}
\nabla_{\mathbf{C}} E(s)= & \phi \nabla_{\mathbf{C}} E_{1}(s)+\tilde{f} \nabla \phi \\
& -(\nabla \phi \cdot \mathbf{T})(\mathbf{T}+\lambda(\nabla I \cdot \mathbf{T}) \nabla I) \tilde{f}^{-1}
\end{aligned}
$$

which is indeed in the normal direction:

$$
\begin{aligned}
& \nabla_{\mathbf{C}} E(s)=\left((\nabla \phi \cdot \mathbf{N}) \tilde{f}-\phi \kappa \tilde{f}^{-1}-\lambda(\nabla I \cdot \mathbf{N})\right. \\
& \left.\cdot\left((\nabla \phi \cdot \mathbf{T})(\nabla I \cdot \mathbf{T})+\phi(\nabla I \cdot \mathbf{T})_{s} \tilde{f}^{-2}\right) \tilde{f}^{-1}\right) \mathbf{N}
\end{aligned}
$$

as expected since the energy (4) is re-parameterization invariant. It is straightforward to check that (9) provides the formula of the gradient of the GAC energy in the particular case $\lambda=0: \nabla_{\mathbf{C}} E(s)=((\nabla \phi \cdot \mathbf{N})-\phi \kappa) \mathbf{N}$. The gradient flow corresponding to the minimization of (4) is

$$
\mathbf{C}_{t}=-\nabla_{\mathbf{C}} E(s)
$$

\subsection{Implementation}

The curve evolution (10) is implemented numerically in the implicit (Eulerian) formulation of Level Set methods [12]. If $u: \Omega \rightarrow \mathbb{R}$ is the function whose zero level set is $\mathbf{C}$ and the curve evolves with a speed in the normal direction $\beta$, $\mathbf{C}_{t}=\beta \mathbf{N}$, the corresponding embedding function evolves as $u_{t}=\beta\|\nabla u\|$ (see [2]). Given an initial contour, we seek for the steady state solution of this equation so that the resulting contour satisfies the first-order optimality conditions (Euler-Lagrange equations $\nabla_{\mathbf{C}} E(s)=0$ ). In our case, $\beta=$ $-\nabla_{\mathbf{C}} E(s) \cdot \mathbf{N}$ and all geometric entities in (9) (e.g., $\left.\mathbf{T}, \mathbf{N}, \kappa\right)$ can be computed in terms of the embedding function $u$ : recall that $\mathbf{N}=-\nabla u /\|\nabla u\|, \mathbf{T}$ is a $90^{\circ}$ rotation of $\mathbf{N}$ and $\kappa=$ $\operatorname{div}(-\mathbf{N})$. We choose as edge indicator function $\phi(\mathbf{x})=(1+$ $\left.\left\|\nabla G_{\sigma} * I\right\|^{2}\right)^{-1}$, where $G_{\sigma}$ is a Gaussian smoothing filter. Using finite differences to approximate derivatives (forward differences in time and central and upwind differences in space for diffusive and advective terms of the speed function, respectively), yields an explicit incremental update scheme for the discrete approximation of $u$.

\section{EXPERIMENTS}

The proposed technique has been tested on medical magnetical resonance images (MRI). We report the results of evolving contours to extract the boundaries of the heart chambers of a dog in Figs. 2 and 3.

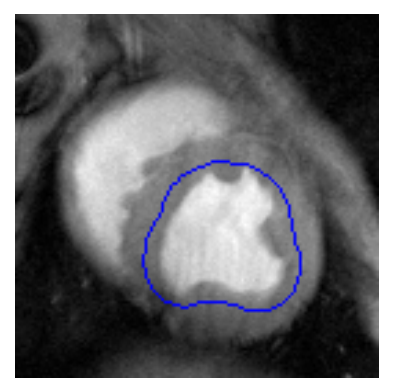

(a) $I(\mathbf{x})$ and initial contour

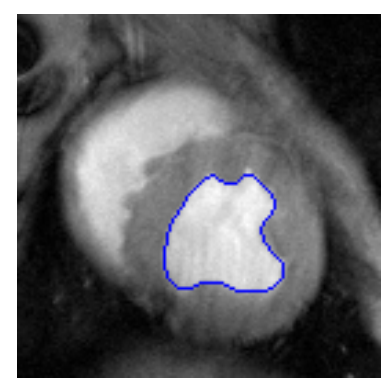

(c) Result of GAC

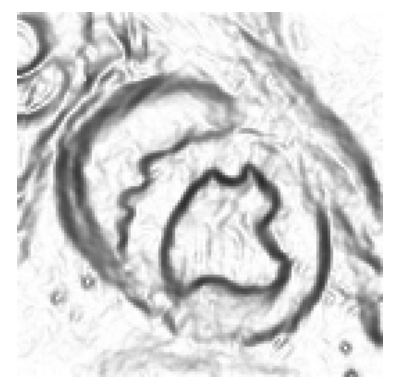

(b) Edge map $\phi(\mathbf{x})$

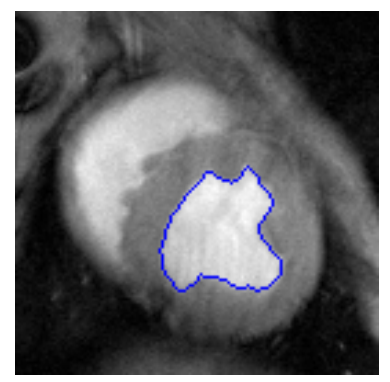

(d) Result of flow (10)
Fig. 2. Contour extraction from MRI heart image.

Experimentally, we have found that $\lambda \in[0.01,1]$ offers a good balance between both penalties in (3) for images with values in the range $[0,255]$. Fig. 2(a) shows the common initialization for the two compared methods. Fig. 2(b) is the edge map $\phi(\mathbf{x})$ of image $I(\mathbf{x})$ in (a). Fig. 2(c) shows the contour obtained by means of the GAC model with edge indicator $\phi$. Finally, Fig. 2(d) shows the result of our proposed technique, the directional GAC model. Both techniques successfully detect the boundary of the left ventricle, however there are subtle differences. The conformal factor due to the edge indicator function $\phi$ has a major contribution to the correct detection of the object boundary. The proposed penalty term $(\mathbf{T} \cdot \nabla I)^{2}$ in directional GAC model (3) sharpens the contour, specially at corners of the boundary of the objects in the image where the GAC model typically shows a rounding off effect. In particular, this is observed in the top right corner of the ventricle in Fig. 2 and it is more evident in the next experiment (Fig. 3), which presents a more challenging target object boundary. 


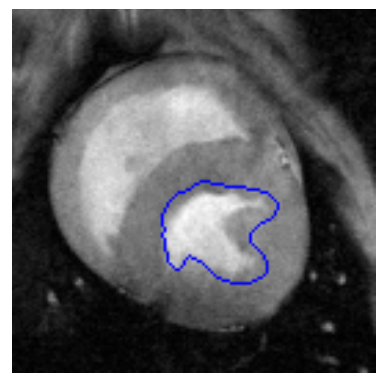

(a) $I(\mathbf{x})$ and initial contour

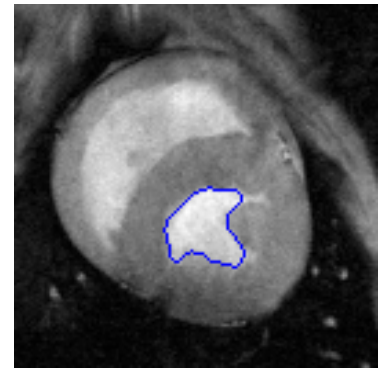

(c) Result of GAC

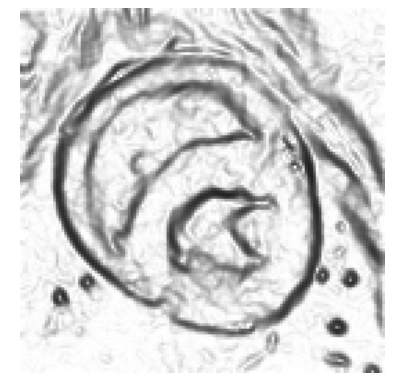

(b) Edge map $\phi(\mathbf{x})$

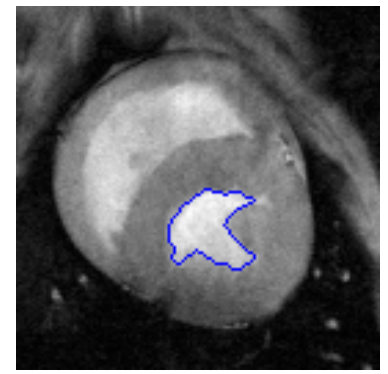

(d) Result of flow (10)
Fig. 3. Contour extraction from MRI heart image.

In Fig. 3(c), the lack of directionality in the regularization within the GAC model refrains the contour from extracting acute edge corners. In Fig. 3(d), the directionality within our model improves the detection capabilities of the active contour.

\section{DISCUSSION}

We have shown how, from geometric local designing principles, the geodesic active contour model (GAC) can be generalized to assimilate directional information of the images while remaining in a Riemannian framework. Hence, the minimizing curves of the proposed model admit the interpretation of being geodesics of a newly defined metric. An edgebased functional and its corresponding gradient descent flow have been presented and tested on MRI images. Our model outperforms the GAC model for the segmentation of objects with sharp boundaries.

A possible research direction to follow up would be the extension of the proposed non-conformal edge-based energy functional to the Sobolev framework [11], which would improve the robustness with respect to the initialization curve, hence mitigating the problem of getting trapped in local minima. Our model could also be extended to the segmentation of surfaces using analogous principles to define an anisotropic area element, etc.

\section{ACKNOWLEDGEMENTS}

We would like to thank Prof. A. Yezzi for the level set software and MRI images used in the experiments.

\section{REFERENCES}

[1] M. Kass, A. Witkin, and D. Terzopoulos, "Snakes: Active contour models," Int. J. Computer Vision, vol. 1, no. 4, pp. 321-331, 1988.

[2] V. Caselles, R. Kimmel, and G. Sapiro, "Geodesic active contours," Int. J. Computer Vision, vol. 22, no. 1, pp. 61-79, 1997.

[3] J. Melonakos, E. Pichon, S. Angenent, and A. Tannenbaum, "Finsler active contours," IEEE Trans. Pattern Anal. Mach. Intell., vol. 30, no. 3, pp. 412-423, 2008.

[4] T.F. Chan and L.A. Vese, "Active contours without edges," Image Processing, IEEE Trans. on, vol. 10, no. 2, pp. 266-277, 2001.

[5] R. Kimmel and A. M. Bruckstein, "Regularized laplacian zero crossings as optimal edge integrators," Int. J. Computer Vision, vol. 53, pp. 225-243, 2003.

[6] A. Vasilevskiy and K. Siddiqi, "Flux maximizing geometric flows," IEEE Trans. Pattern Anal. Mach. Intell., vol. 24, pp. 1565-1578, 2002.

[7] C. Harris and M. Stephens, "A combined corner and edge detector," in Alvey Vision Conference, 1988, vol. 15, pp. 147-151.

[8] J. Weickert, Anisotropic Diffusion in Image Processing, ECMI Series, Teubner-Verlag, Stuttgart, Germany, 1998.

[9] A. Yezzi, "Modified curvature motion for image smoothing and enhancement," IEEE Trans. on Image Processing, vol. 7, no. 3, pp. 345-352, 1998.

[10] N.A. Sochen, R. Kimmel, and R. Malladi, "A general framework for low level vision," IEEE Trans. on Image Processing, vol. 7, no. 3, pp. 310-318, 1998.

[11] G. Sundaramoorthi, A. Yezzi, and A. Mennucci, "Sobolev active contours," Int. J. Computer Vision, vol. 73, no. 3, pp. 345-366, 2007.

[12] S. Osher and J. A. Sethian, "Fronts propagating with curvature-dependent speed: algorithms based on hamilton-jacobi formulations," J. Comput. Phys., vol. 79, pp. 12-49, 1988. 